\title{
Los conocimientos faunísticos en el estudiantado de escuelas rurales: Una guía para su reconocimiento en la clase de ciencias naturales
}

\author{
Faunal knowledge of students in rural schools: a guide for their recognition in science class
Os conhecimentos faunísticos dos estudantes das escolas rurais: uma guia para seu reconhecimento na classe de ciência

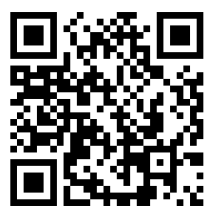

\author{
Rubinsten Hernández-Barbosa ${ }^{1}$ \\ Universidad Autónoma de Colombia \\ Bogotá, Colombia \\ rhbjd@hotmail.com \\ http://orcid.org/0000-0002-5595-5344
}

Recibido $\cdot$ Received $\cdot$ Recebido: 23 / 01 / 2017
Corregido $\cdot$ Revised $\cdot$ Revisado: 09 / 12 / 2017
Aceptado $\cdot$ Accepted $\cdot$ Aprovado: 07/ 02 / 2018

\begin{abstract}
Resumen: Este texto tiene por objetivo describir una propuesta metodológica para identificar, clasificar y organizar los conocimientos faunísticos que tiene el estudiantado de las escuelas del sector rural. El trabajo se desarrolló con 20 estudiantes de sexto grado, de edades entre 11 y 14 años, de una escuela rural del Departamento de Cundinamarca, Colombia. A través de cinco tipos de actividades, expresaron, de diferentes maneras, sus conocimientos sobre los animales de la región. Esta información fue organizada, categorizada y sistematizada en tablas, las cuales son el resultado del análisis de la información suministrada por el estudiantado. Es una posibilidad de trabajo escolar que favorece el reconocimiento y la valoración de los conocimientos tradicionales y ancestrales y su incorporación a la dinámica de la enseñanza de las ciencias naturales, como una forma de crear "puentes" entre estos saberes y el conocimiento científico escolar. Es una propuesta que, entre otras cosas, favorece el desarrollo de actitudes más positivas hacia la ciencia misma, motiva al estudiantado a hacer preguntas, a reconocer la importancia del contexto cultural y a reconocerse como parte de un sistema biocultural.
\end{abstract}

Palabras claves: Conocimiento científico escolar; currículo; enseñanza de las ciencias; etnobiología.

\footnotetext{
${ }^{1}$ Culminó estudios de Licenciatura en Química y Biología en la Universidad INCCA de Colombia. Realizó estudios de Maestría en Biología en la Pontificia Universidad Javeriana y de Especialización en Pedagogía de Proyectos, Doctor en Educación de la Universidad Distrital Francisco José de Caldas. A lo largo de su ejercicio docente ha participado en seminarios y congresos con énfasis en educación y pedagogía. Actualmente se desempeña como docente de tiempo completo en la Universidad Autónoma de Colombia en el programa de Maestría en Didáctica de las Ciencias.
} 
doi: http://dx.doi.org/10.15359/ree.22-2.1

URL: http://www.una.ac.cr/educare

CORREO: educare@una.cr

\begin{abstract}
This text aims to describe a methodological proposal to identify, classify, and organize the faunistic knowledge of students of rural schools. The research was conducted with twenty sixth graders from a rural school in the Department of Cundinamarca, Colombia. Through five types of activities, they expressed, in different ways, their knowledge about the animals of the region. The information collected was organized, categorized, and systematized in tables; these tables resulted from the analysis of the information the students provided. It is a possibility of school work that favors the recognition and valuation of the traditional and ancestral knowledge, and its incorporation to the dynamics of the teaching and learning of the Natural Sciences as a way to create "bridges" between that knowledge and the scholarly scientific knowledge. It is a proposal that, among other things, favors the development of more positive attitudes toward science itself, motivates students to ask questions, to recognize the importance of the cultural context, and to recognize themselves as part of a biocultural system.
\end{abstract}

Keywords: School science knowledge; curriculum; teaching science; ethnobiology.

Resumo: O objetivo deste trabalho é descrever uma metodologia para identificar, classificar e organizar os conhecimentos faunísticos dos estudantes de escolas da área rural. O trabalho foi realizado com 20 estudantes da sexta série, com idades entre 11 e 14 anos, de uma escola rural no Departamento de Cundinamarca, Colômbia. Através de cinco tipos de atividades, eles expressaram de diferentes maneiras seus conhecimentos sobre os animais da região. Esta informação foi organizada, categorizada e sistematizada em tabelas, que são o resultado da análise das informações fornecidas pelos estudantes. É uma possibilidade de trabalho escolar que promove o reconhecimento, a valorização e incorporação dos conhecimentos tradicionais e ancestrais a dinâmica do ensino das ciências naturais, como uma forma de criar "pontes" entre estes saberes e o conhecimento científico escolar. É uma proposta que, entre outras coisas, promove o desenvolvimento de atitudes positivas em relação à própria ciência, motiva os estudantes a perguntar, a reconhecer a importância do contexto cultural e reconhecer-se como parte de um sistema biocultural.

Palavras-chave: Conhecimento científico escolar; currículo; ensino das ciências; etnobiologia.

\title{
Introducción
}

La relación de los animales con el ser humano y su entorno data de tiempos remotos, desde el mismo comienzo de la humanidad, y se manifiesta de muchas formas. Además de su patrimonio biológico natural, los animales han sido inspiración de poetas, escritores y cantores de música folclórica, entre otras expresiones artísticas; se ha establecido, de esta manera, su importancia como patrimonio zoocultural, el cual se refleja en diferentes expresiones materiales e inmateriales como la pintura, monumentos, artesanías, cerámica, refranes y música, entre otros (Clavijo, 2009).

El conocimiento faunístico que el estudiantado del sector rural tiene puede estar enmarcado dentro de cualquiera de las expresiones señaladas anteriormente, su identificación y reconocimiento en la escuela, y particularmente desde la clase de ciencias naturales, ofrece una gran posibilidad de diálogo entre estos saberes y el conocimiento científico escolar. 
En ese sentido, una enseñanza de las ciencias con enfoque contextual/sociocultural puede favorecer, entre otras cosas:

- Identificación y reconocimiento de la riqueza y diversidad tanto animal como vegetal,

- formas y propuestas para cuidar el medio ambiente y los ecosistemas,

- generación de emociones positivas hacia su entorno natural y social (Hernández, 2017, p. 284).

Considerando lo anterior, en este texto se describen formas diversas que puede usar el personal docente de ciencias naturales para identificar, clasificar y organizar los conocimientos que el estudiantado del sector rural tiene sobre los animales de la región donde se inserta la institución escolar, con el objetivo de crear puentes de diálogo, intercambio de ideas y comunicación entre este tipo de conocimientos y los que se establecen en los currículos oficiales o de los textos escolares; los cuales, la mayoría de las veces, se encuentran organizados y estructurados de manera lineal y rígida, y que el personal docente de ciencias naturales (biología, química y física) aborda, obedeciendo más a una lógica curricular propuesta por los entes ministeriales o editoriales, en la que presentan una hegemonía y sobrevaloración de los conocimientos científicos, olvidando los conocimientos cotidianos y tradicionales del estudiantado sobre su contexto natural (Hernández, 2014). La propuesta que se trae a consideración es el resultado del trabajo desarrollado por el autor ${ }^{2}$ en un modelo de intervención y acompañamiento a una docente de ciencias naturales del sector rural. El propósito es reconocer y valorar dichos conocimientos, los cuales pueden ser incorporados en el escenario educativo, más concretamente en las dinámicas de las clases de ciencias naturales.

\section{Referentes teóricos}

Los aspectos abordados en la introducción conllevan a establecer la necesidad de comprender, tal y como anota Delors (1996), que la educación debe encaminarse a cumplir el conjunto de tareas que le son propias, esta debe estructurarse en cuatro pilares: aprender a aprender, aprender a hacer, aprender a ser y aprender a vivir juntos. Si bien estos aspectos se ven como cardinales, para su consolidación dentro de un contexto escolar determinado, en el que sus agentes establecen diferentes tipos de relaciones, y particularmente con el conocimiento de las ciencias, es necesario promover acciones que permitan la construcción de modelos de enseñanza

\footnotetext{
${ }^{2}$ El modelo de intervención y acompañamiento hizo parte de las actividades desarrolladas por el autor para dar cumplimiento a uno de los objetivos de su tesis doctoral El contexto cultural en las prácticas educativas de profesores de ciencias del sector rural: Perspectivas para el modelo de formación por cambio didáctico, la cual realizó con la dirección del Dr. Carlos Javier Mosquera Suárez, en la Universidad Distrital Francisco José de Caldas, Bogotá, Colombia.
} 
doi: http://dx.doi.org/10.15359/ree.22-2.1

URL: http://www.una.ac.cr/educare

CORREO: educare@una.cr

de esta misma, donde se tengan presentes las dimensiones psicológica, epistemológica, didáctica y contextual, que conlleve a la mejora de las prácticas educativas del profesorado de ciencias, a partir de procesos autorregulatorios de sus propias acciones como docentes.

Estas consideraciones, contextualizadas en un país como Colombia, de contrastes económicos y de oportunidades, invitan a repensar la educación como camino fructífero para disminuir las brechas entre las diferentes comunidades que lo conforman, implican establecer un compromiso social que conduzca al desarrollo de reformas educativas donde los diferentes sectores se responsabilicen a corto, mediano y largo plazo para intervenir en la mejora de las condiciones para una educación digna y de calidad, sin distingo de clases sociales o de posibilidades económicas de quienes aprenden.

Setrata, entonces, de pensary proyectaruna sociedad másjusta e incluyente, especialmente considerando la condición pluriétnica y pluricultural del país. Esta forma de interpretar el papel de la educación direcciona a suponer la necesidad de pensar que la apropiación y construcción de los diferentes tipos de conocimientos depende, entre otros aspectos, de los valores, creencias, intereses y necesidades de las personas (Molina, Mojica y López, 2005). Es así como en el marco de la enseñanza de las ciencias naturales Molina, Martínez, Mosquera y Mojica (2009) han identificado y caracterizado cuatro perspectivas.

1. Los universalistas. En este grupo se incluyen quienes piensan que la ciencia tiene un carácter universal y, por ende, su enseñanza.

2. Los multiculturalistas. A esta categoría pertenecen quienes, además del conocimiento científico, reconocen otros tipos de conocimientos como el traditional, ecological knowledge (TEK), el cual consideran debe incorporarse en los planes de estudio para la enseñanza de las ciencias.

3. Los pluralistas epistemológicos. A esta perspectiva pertenecen quienes señalan que el conocimiento científico es solo una forma de conocimiento, admiten y destacan la existencia de otros tipos de conocimientos, de igual importancia.

4. Los interculturalistas. Los partidarios de esta perspectiva destacan la importancia que deben tener los conocimientos ancestrales y tradicionales para la enseñanza de las ciencias naturales. Es así como Jegede (1995) plantea la necesidad de considerar para la enseñanza de las ciencias dos contextos culturales: uno relacionado con la cultura del estudiantado, y el otro, con el contexto cultural de la ciencia occidental, que es la que prima en la enseñanza de las ciencias naturales. Estos dos tipos de conocimientos, que tienen epistemologías, objetivos, formas de producción, validación y socialización diferentes, pueden entrar en relación a través de puentes George (2001), que sirven para transitar de un contexto a otro. 
En Colombia, y en la mayoría de países de América Latina, ha dominado, en la enseñanza de las ciencias, una mirada universalista de las ciencia, trayendo como consecuencia, en muchos casos, el no tener en cuenta, incluso menospreciar e invisibilizar, otros tipos de conocimientos. Esta sobrevaloración de los conocimientos científicos, la mayoría de las veces va en detrimento de los saberes locales, tradicionales y ancestrales, entre otros (Baptista, 2007), lo cual, a su vez, se refleja en el menoscabo también de los conocimientos que la niñez tiene sobre el mundo natural (Lopes, 1999). Estos aspectos son una muestra clara de desigualdad e inequidad.

Es de vital importancia que en la educación se tomen en cuenta enfoques culturales, que estén orientados a valorar la importancia del contexto y de la diversidad cultural para la enseñanza de las ciencias, lo cual estaría en concordancia con lo establecido en la Constitución de 1991 (Gobierno de Colombia, Asamblea Nacional Constituyente, 1991), en la que se reconoce a Colombia como un país megadiverso, pluriétnico y pluricultural. Lo anterior conlleva, inevitablemente, a pensar en el papel y compromiso de la escuela y del profesorado, particularmente de ciencias naturales, pues es el llamado, desde la institución escolar y en comunión con la comunidad, a dotar de sentido y significado a los aspectos relacionados con la pluriculturalidad, la interculturalidad, el contexto, la diversidad biológica y cultural. Es desde esta mirada y marco conceptual que se desarrolló parte del trabajo que a continuación se describe.

\section{Metodología}

Esta propuesta es el resultado del trabajo de aula que desarrolló el autor con una docente de ciencias naturales en la Institución Educativa Rural Departamental San Lorenzo, a dos horas y media de la capital Bogotá, Colombia, adscrita al municipio de Fomeque, Cundinamarca, en el marco de una de las etapas de su investigación doctoral. En la jurisdicción de este municipio se localiza el Parque Natural Chingaza, que tiene una extensión de 76.452 hectáreas, es una fuente hídrica de importancia para la región, pues allí nacen varios ríos; y es una zona de gran diversidad biológica tanto vegetal como animal. La experiencia de aula se desarrolló con 20 estudiantes de sexto grado, de edades entre los 11 y 14 años.

Es importante que las personas lectoras comprendan que lo que aquí se propone es un camino, una ruta, una guía, y que precisamente es el equipo docente quien, retomando los conocimientos faunísticos del estudiantado y teniendo presente el contexto cultural donde estos toman sentido y significado, establezca formas y medios para incorporarlos, valorarlos y considerarlos en la enseñanza de las ciencias naturales. De esta manera es posible construir puentes entre los diferentes tipos de conocimiento y considerar nuevas perspectivas en este campo.

A continuación, se describen las actividades que se proponen para identificar, clasificar y organizar el conocimiento faunístico que los niños y las niñas de las escuelas rurales tienen sobre los animales propios de su región, con el fin de reconocerlos, valorarlos e incluirlos en la estructura del plan de estudios de la enseñanza de las ciencias naturales. Aunque en 
doi: http://dx.doi.org/10.15359/ree.22-2.1

URL: http://www.una.ac.cr/educare

CORREO: educare@una.cr

la experiencia se usaron todas las actividades, la elección de algunas de ellas, por parte del personal docente, depende de factores y recursos particulares de cada institución: ubicación geográfica, materiales, tiempo, equipos (cámara fotográfica, computador, fotocopiadora) e incluso permisos institucionales y familiares para el caso del trabajo de campo.

Dibujo. En el dibujo, como una forma de representación gráfica, que es más antigua que el lenguaje escrito, las culturas exteriorizan determinadas maneras de ver, sentir y entender el mundo (Silveira, 1997, citado por Baptista, 2007). En una primera parte se les puede solicitar que hagan dibujos de los animales que conocen. También se les puede pedir que en el dibujo ubiquen las partes con su respectiva denominación. Este ejercicio permite identificar parte de la fauna de la región y su morfología externa. Otro ejercicio consiste en pedirles que, de una lista de animales de la zona, seleccionen un animal y lo dibujen. La elección puede estar permeada por varios factores: cercanía del animal, sentimientos y emociones que le genera a la niñez, y aspectos físicos que llaman la atención al estudiantado: formas, colores, tamaños y comportamientos, entre otros. Esta actividad tiene dos ventajas; la primera, a la mayoría de los niños y las niñas les gusta dibujar, y la segunda, se convierte para el equipo docente en un insumo inicial para ser usado como material de análisis.

El análisis del dibujo permite conocer no solo la morfología externa del animal, también las partes que el estudiantado conoce y las expresiones que usan para identificarlas, así como referir el hábitat, en ocasiones el nicho, y las relaciones que establecen con otros organismos y factores abióticos. Sin embargo, el dibujo por sí solo no es suficiente, especialmente cuando se representa el animal "únicamente", pues solo permite inferir un conocimiento anatómico externo del animal, dejando de lado otros aspectos que aquí se describen y que surgieron del trabajo realizado.

Realización de textos orales y escritos. Con los dibujos se puede hacer una presentación o una exposición. En cualquiera de los casos, se les puede solicitar a los niños y a las niñas que hagan una descripción oral o escrita del animal que han seleccionado. Una pregunta orientadora puede ser: ¿Qué sabes sobre el animal que escogiste? Teniendo en cuenta lo expuesto por los niños y las niñas, lo cual es el reflejo, en parte, de sus conocimientos, en un segundo momento, y dependiendo del aspecto sobre el cual se quiere hacer énfasis, otras preguntas que se pueden proponer son las siguientes:

- ¿Por qué escogiste ese animal?

- ¿Cuáles son las características físicas del animal que seleccionaste?

- ¿Cómo es el sitio dónde vive el animal? ¿Cuál es el hábitat del animal que seleccionaste?

- ¿Con qué otros animales se relaciona? ¿Cómo se relaciona?

- ¿De qué se alimenta el animal que seleccionaste? ¿Cómo consigue el alimento? 
- ¿Cómo se reproduce? ¿Cuántas crías tiene? ¿Se pueden diferenciar los machos de las hembras?

- ¿Cuál es la importancia del animal que seleccionaste en la naturaleza?

- ¿De qué manera se relaciona con los habitantes de la región?

- ¿Cuáles son los usos que le dan al animal?

La entrevista. Como complemento a las actividades anteriores, se propone la entrevista, actividad que llama la atención del estudiantado, pues la idea es que sean ellos mismos los que entrevisten a miembros de la comunidad (padres, madres, abuelos, abuelas, profesorado, vecindario, estudiantado de grados superiores). Este ejercicio se desarrolla en cinco momentos. En todos ellos el acompañamiento y colaboración del profesorado es importante. 1. Selección del animal, 2. Elección de la persona entrevistada, 3. Planeación y organización de las categorías y preguntas, 4 . Desarrollo de la entrevista y 5 . Socialización del ejercicio.

Trabajo de campo. El trabajo de campo y las salidas pedagógicas suelen ser llamativas y enriquecedoras para el estudiantado, pues además de ser motivadoras, favorecen, en diferentes grados, estímulos visuales, auditivos, táctiles y olfativos, con respecto a la actividad que se propone. Es una opción que puede usar el equipo docente, incluso en combinación con las actividades anteriores, pues el dibujo y la descripción lo pueden hacer al aire libre y si es posible observando el animal escogido. Sabemos que no en todos los casos es posible, y que la observación de algunos animales, por su propio comportamiento y hábitat, no es fácil. Por ello se propone la actividad del álbum fotográfico. En la salida de campo también se puede hacer la entrevista. Se sugiere hacer un trabajo fotográfico, que luego pueda ser expuesto y usado en la actividad que se describe a continuación.

Álbum fotográfico. Es el resultado de la selección de las fotografías por parte del docente o la docente, quienes, con base en la información del estudiantado, o de la propia consulta que hacen sobre los animales presentes en la zona, organiza el álbum. Además de la descripción, dependiendo de los objetivos del equipo docente, el álbum se puede organizar de varias formas: vertebrados, invertebrados, insectos, mamíferos, o los seleccionados por el estudiantado en las primeras actividades. Para su elaboración se sugiere usar una impresora de alta resolución, a color y dividir el cartel en dos partes. En la primera, se ubica el dibujo del animal únicamente; $y$, en el segundo, el animal en su medio ambiente natural o nicho ecológico.

También se puede, si es necesario, presentar fotografías que representen la anatomía interna. El énfasis del álbum lo establece el personal docente, dependiendo de los objetivos que se propongan. El objetivo es que, mediante la observación de las fotos, estas sean motivadoras para que el estudiantado exprese sus conocimientos, de distinto orden, sobre los animales, como también sus sentimientos y usos entre otros aspectos. La utilización de este recurso debe 
doi: http://dx.doi.org/10.15359/ree.22-2.1

URL: http://www.una.ac.cr/educare

CORREO: educare@una.cr

complementarse con preguntas que el docente o la docente puede hacer, aspecto descrito previamente. Como complemento al álbum se propone también la observación de videos, los cuales pueden estar relacionados con animales de la región geográfica donde se inserta la escuela.

Ficha de trabajo de organización de la información. Se emplea para recopilar y organizar la información de acuerdo con categorías previamente establecidas, las cuales pueden emerger de los conocimientos que expresan y evidencian en los dibujos o en la presentación de fotos o videos sobre los animales. Estas fichas las elabora el equipo docente o se pueden ir elaborando con el estudiantado. Según Bogdan y Biklen (1994, citado por Baptista, 2004), las categorías son una forma de clasificar los datos descriptivos que favorece luego su interpretación. Estas fichas, en muchos casos, facilitan el trabajo posterior, como la organización y secuenciación de actividades de enseñanza, la publicación de un artículo, la sistematización de una investigación y la socialización de los resultados a la comunidad, entre otros. En este caso son usadas con un doble propósito, uno, reconocer el conocimiento que el estudiantado tiene sobre los animales, y dos, para organizar y estructurar las actividades que permitan conexión con el programa curricular en ciencias naturales establecido para la institución escolar.

Las fichas de trabajo se pueden hacer en cartulinas o también diseñarlas en un programa de computador y hacer su propia base de datos. A continuación, se propone un modelo de ficha para obtener información sobre los conocimientos que tiene el estudiantado de educación básica de las zonas rurales, población indígena o grupos culturalmente diversos sobre los animales de una región o sitio determinado. También puede servir como insumo para abordar y organizar una investigación etnozoológica. La ficha se organiza por categorías y subcategorías; también se describen los aspectos que pueden abarcar en cada una de ellas.

Como toda ficha debe llevar una identificación con una serie de datos que el docente o la docente establece con anterioridad. Algunos datos, como ejemplo, se muestran en la Tabla 1.

Tabla 1: Identificación de la ficha

\begin{tabular}{l|l}
\hline Nombre del estudiante & \\
\hline Grado-curso & \\
\hline Fecha: año-mes-día & \\
\hline Zona de recolección de información & \\
\hline No de ficha & \\
\hline
\end{tabular}

Nota: Elaboración propia. 
En cuanto a la ubicación del animal, es importante señalar una región biogeográfica, y para ello se sugiere hacer un mapa, que puede ser elaborado por el propio estudiantado, o tomando una copia o croquis ampliado de internet sobre el cual señalar los lugares posibles de hábitat. Para cada animal se sugiere completar la información de la Tabla 2.

Tabla 2: Región o zonas de localización del animal

Ubicación biogeográfica Descripción y condiciones del sitio o ecosistema donde se puede encontrar el animal. Es importante hacer una descripción detallada de los lugares y cómo se puede acceder a ellos. En ese sentido el mapa es un buen recurso.

Nota: Elaboración propia.

En la Tabla 3 se discrimina la información que debe organizarse para cada uno de los organismos que se ha establecido estudiar en relación con su denominación y taxonomía.

Tabla 3: Nombre del animal y clasificación

\begin{tabular}{ll}
\hline Nombre común & $\begin{array}{l}\text { Nombre común con el que se conoce el animal en la región o } \\
\text { zona geográfica, para el caso de grupos indígenas se establece } \\
\text { también el nombre en el dialecto de la etnia. }\end{array}$ \\
\cline { 2 - 3 } & $\begin{array}{l}\text { En algunos casos es posible que el nombre de la lengua nativa } \\
\text { corresponda con el género del grupo de organismos al cual } \\
\text { pertenece el animal, pues puede darse que varias especies se } \\
\text { identifiquen con un mismo nombre o lo contrario. }\end{array}$ \\
\hline \multirow{3}{*}{ Clasificación } & $\begin{array}{l}\text { Se parte de las descripciones y aspectos particulares que la } \\
\text { comunidad tiene para clasificarlos, los cuales se extienden al } \\
\text { estudiantado, quienes organizan grupos, generalmente teniendo } \\
\text { en cuenta los aspectos anatómicos externos, y a medida que } \\
\text { se desarrolla la actividad van surgiendo clasificaciones mucho }\end{array}$ \\
& $\begin{array}{l}\text { más específicas. Este aspecto puede ser uno de los últimos por } \\
\text { abordar con el estudiantado. Es importante decir que los criterios } \\
\text { de clasificación pueden ser guiados por el equipo docente, y } \\
\text { van a depender, entre otros factores, de las características de la } \\
\text { investigación realizada, el tema que se esté abordando, las edades } \\
\text { y los objetivos específicos del trabajo escolar a nivel curricular. }\end{array}$ \\
\hline
\end{tabular}

Nota: Elaboración propia.

En la Tabla 4 se señalan los aspectos anatómicos, tanto internos como externos, que pueden ser descritos por el estudiantado y, por lo tanto, objeto de estudio. La anatomía se encarga de estudiar las estructuras macroscópicas de los seres vivos, aspecto que abarca, entre otras cosas, la forma, la ubicación y la disposición de los órganos que conforman un sistema. Es importante anotar que los aspectos anatómicos no se pueden desligar de la función. 
doi: http://dx.doi.org/10.15359/ree.22-2.1

URL: http://www.una.ac.cr/educare

CORREO: educare@una.cr

Tabla 4: Aspectos anatómicos

\begin{tabular}{cl}
\hline \multirow{2}{*}{ Anatomía externa } & $\begin{array}{l}\text { Hace referencia a los aspectos visibles y que caracterizan a un grupo de } \\
\text { organismos, por ejemplo, la presencia de cuernos, forma de la nariz, tipo de } \\
\text { dientes, orejas, picos, bigotes, número de patas, color, manchas, pelos, tamaño } \\
\text { y posibles diferencias entre los machos y las hembras. Estos aspectos también } \\
\text { pueden ser usados para trabajar conceptos y aspectos de genética. }\end{array}$ \\
\cline { 2 - 4 } Anatomía & $\begin{array}{l}\text { Hace referencia a las partes internas del organismo, las cuales pueden ser } \\
\text { identificadas y relacionadas a su vez con una función fisiológica. Ejemplo: } \\
\text { corazón, venas, capilares, arterias y función y características de la sangre, para } \\
\text { el caso de la circulación. }\end{array}$ \\
\hline
\end{tabular}

Nota: Elaboración propia.

La fisiología estudia el funcionamiento de los órganos que componen un organismo y el cuerpo en su conjunto. Se puede hacer a diferentes niveles: celular, órganos, sistemas y organismo completo. En la Tabla 5 se establecen los aspectos fisiológicos y tópicos que se pueden considerar en cada uno de ellos.

Tabla 5: Aspectos fisiológicos

\begin{tabular}{cl}
\hline Nutrición & $\begin{array}{l}\text { Se tiene en cuenta el tipo de alimento y la manera como el animal lo obtiene. } \\
\text { También se pueden analizar aspectos comola ubicación en las cadenas alimenticias } \\
\text { y tipo de relación simbiótica. }\end{array}$ \\
\hline Respiración & $\begin{array}{l}\text { Se considera la manera como respiran y el papel que juega el aire y el oxígeno en } \\
\text { dicha función. También se puede considerar el tipo de respiración, los órganos } \\
\text { comprometidos y su relación con otros sistemas. }\end{array}$ \\
\hline Fisiología & $\begin{array}{l}\text { Entre los aspectos que pueden agruparse en esta categoría están: la sangre, su } \\
\text { colory funciones; presencia o ausencia de sangre en algunos organismos; órganos } \\
\text { comprometidos con la función circulatoria y los sistemas de conducción: vasos } \\
\text { capilares, venas y arterias. }\end{array}$ \\
\cline { 2 - 3 } Reproducción & $\begin{array}{l}\text { El conocimiento del estudiantado en esta categoría puede abarcar diferencias entre } \\
\text { machos y hembras, cortejo, número de crías, cuidado y zona de desarrollo de las crías. }\end{array}$ \\
\hline Excreción & $\begin{array}{l}\text { Formas como eliminan los residuos del metabolismo, entre los aspectos que } \\
\text { pueden señalar están: presencia y tipo o formas de excreción, lugar de depósito } \\
\text { de las excreciones, olor, tamaño y aspectos físico. También pueden señalar el uso } \\
\text { de las excreciones, si es el caso, por el ser humano. }\end{array}$ \\
\hline
\end{tabular}

Nota: Elaboración propia.

\begin{tabular}{l|l}
\hline 10 & Rubinsten Hernández-Barbosa
\end{tabular}

Los artículos de la Revista Electrónica Educare del Centro de Investigación y Docencia en Educación de la Universidad Nacional, Costa Rica, se comparten bajo términos de la Licencia Creative Commons: Reconocimiento, No Comercial, Sin Obra Derivada 3.0 Costa Rica. Las autorizaciones adicionales a las aquí delimitadas se pueden obtener en el correo: educare@una.cr 
La ecología es una rama de la biología, de carácter interdisciplinario, que estudia las interacciones entre los organismos vivos y el entorno natural en el que habitan (Margalef, 1998). Para el caso del grupo con quienes se desarrollaron las diversas formas de reconocer el conocimiento faunístico que se propone en este texto, este fue el tipo de conocimiento más amplio y el que emergió en los primeros momentos de diálogo con el estudiantado. La Tabla 6 categoriza los aspectos ecológicos.

Tabla 6: Aspectos ecológicos

\begin{tabular}{|c|c|c|}
\hline \multirow{9}{*}{ Ecología } & Hábitat & $\begin{array}{l}\text { Descripción que tiene que ver con el nicho de cada animal, factores bióticos } \\
\text { y abióticos. Pueden describir espacios específicos en términos de cuevas, } \\
\text { nidos, madrigueras, ríos y hojas de las plantas, entre otros. }\end{array}$ \\
\hline & Comportamiento & $\begin{array}{l}\text { Descripción etológica del animal: agresivo, manso, activos, maneras de } \\
\text { relacionarse con los congéneres y de otras especies. También en su relación } \\
\text { con el ser humano. }\end{array}$ \\
\hline & Reproducción & $\begin{array}{l}\text { Los conocimientos del estudiantado pueden estar relacionados con las } \\
\text { épocas de apareamiento, periodos de celo, formas de cortejo y número de } \\
\text { crías, entre otros. En algunos casos pueden mencionar sistemas de criaderos, } \\
\text { por ejemplo, de peces y gallinas. Esto lo pueden relacionar con sistemas } \\
\text { productivos y economía de la región. }\end{array}$ \\
\hline & Ciclo de vida & $\begin{array}{l}\text { Periodos de vida en años, meses, días y horas. Características físicas de un } \\
\text { animal joven, de un animal viejo, causas de la muerte; en fin, características } \\
\text { propias de cada etapa del ciclo de vida y sus respectivos cambios. }\end{array}$ \\
\hline & Alimentación & $\begin{array}{l}\text { Descripción del tipo de alimento, tiempos, formas y maneras de consecución } \\
\text { del alimento. Relaciones morfológicas del animal y el tipo de alimentación. }\end{array}$ \\
\hline & Desplazamiento & $\begin{array}{l}\text { La manera como los organismos se desplazan, épocas en que lo hacen, } \\
\text { las causas que pueden generar un desplazamiento y la relación con las } \\
\text { estructuras físicas tanto internas como externas. }\end{array}$ \\
\hline & Tiempo de actividad & $\begin{array}{l}\text { Periodos de actividad: nocturnos o diurnos. También puede darse el caso de } \\
\text { organismos vespertinos. }\end{array}$ \\
\hline & Sonidos & $\begin{array}{l}\text { Descripción, y denominación de los sonidos que el animal produce: ladridos, } \\
\text { Ilantos, gritos y aullidos. Algunos de ellos son propios de una clase de animal. } \\
\text { Tiempos y condiciones en los que se produce el sonido y su significado. }\end{array}$ \\
\hline & Huellas & $\begin{array}{l}\text { Las marcas específicas de un animal, uso e importancia de las huellas. Se } \\
\text { pueden comparar y establecer su uso e importancia para el ser humano. }\end{array}$ \\
\hline
\end{tabular}

Nota: Elaboración propia. 
doi: http://dx.doi.org/10.15359/ree.22-2.1

URL: http://www.una.ac.cr/educare

CORREO: educare@una.cr

En los conocimientos tradicionales y ancestrales que el estudiantado del sector rural ha adquirido sobre la fauna de una región se destacan también aquellos que tienen que ver con características y atributos no físicos como las emociones, pues en algunos casos los niños y las niñas les atribuyen emociones como alegría, rabia, miedo, incluso vergüenza a los animales. También tienen conocimientos relacionados con la manera cómo los animales se comunican: canto, ladrido, maullido, aullido, en algunos casos la propia habla, como en el caso de los loros. No solo se pueden caracterizar sino también establecer su importancia para el grupo al que pertenece un animal específico. En algunas comunidades es sugestivo e interesante para la niñez realizar comparaciones con algunos atributos que se dan en los seres humanos. Un ejemplo de ello es la capacidad cognitiva para referirse a si un animal es inteligente, entiende por ejemplo lo que le dicen y saben hacer cosas, por esta misma capacidad.

Además, en algunas comunidades, no solo las indígenas, a algunos animales les atribuyen poderes mágicos, espirituales o sobrenaturales; también a una gran diversidad de animales les atribuyen una capacidad tecnológica, la cual se refiere a la acción que algunos animales hacen cuando construyen cuevas, nidos, madrigueras, incluso herramientas. También puede usarse este tipo conocimiento para reflexionar sobre la intervención humana en los diferentes ecosistemas.

Otro aspecto que puede ser explorado por el personal docente, a través de diferentes actividades, es el uso que las comunidades les dan a los animales, el cual se puede categorizar y relacionar con los conocimientos que son transmitidos de generación en generación, y con la experiencia particular del estudiantado. Por ejemplo, el uso de los animales puede estar en las siguientes condiciones: alimento, comercio, mascotas, curativo y preventivo de enfermedades, transporte y plaga, entre otros. Algunas de estas categorías también pueden ser objeto de reflexión sobre el tráfico de fauna y los animales en vía de extinción. También pueden ser objeto de análisis los usos de los animales que están directamente ligados, para el caso de las tribus indígenas, especialmente para los ritos (Costa, Santos y Vargas, 2009).

\section{Resultados, análisis y discusión}

Esta propuesta se genera como iniciativa para contribuir al proceso de formación continuada de docentes de ciencias naturales, particularmente del sector rural, mediante su aproximación, reconocimiento y valoración de la importancia del contexto cultural, y todos los aspectos que este abarca en el desarrollo del ejercicio profesional docente. Lo anterior en concordancia con los nuevos discursos que destacan la importancia de comprender que la educación en general debe interpretarse en un contexto particular, y que la construcción, apropiación, validación y formas de socialización de los diferentes tipos de conocimientos, depende de los valores y creencias, intereses y necesidades, entre otros aspectos, de los miembros de una comunidad (Enciso y Molina, 2009). 
La propuesta parte de la interlocución, reflexión y análisis que el autor hace entre elementos conceptuales teóricos y una posibilidad del trabajo en el aula del personal docente de ciencias naturales del sector rural. En ese sentido, son varios los aspectos que se consideran en este apartado. Por un lado, el reconocimiento y uso del contexto cultural del estudiantado, de la comunidad y del personal docente mismo a la hora de diseñar el currículo de ciencias naturales. Una de las formas en que se ponen en evidencia aspectos del contexto cultural es, precisamente, a través de los conocimientos alternativos, los cuales es necesario identificar, caracterizar y organizar, para tomarlos como punto de encuentro y de diálogo con los conocimientos científicos escolares que cada docente debe desarrollar como parte de su responsabilidad institucional y social.

Es a partir de la organización que hace el personal docente de ciencias naturales del sector rural sobre los conocimientos alternativos que sus estudiantes tienen sobre la fauna de su región la que le permite y favorece el diseñar unidades, secuencias y estrategias didácticas, donde las actividades que se proponen, no solo sean divertidas, sino que sean motivadoras, que propicien y generen un puente de comunicación entre dichos conocimientos y los conocimientos científicos escolares, los cuales desarrolla cada docente a partir de teorías, modelos y conceptos científicos, con el fin de generar aprendizajes significativos. Es de entenderse que, si bien esta propuesta se hace específicamente para los animales, se puede extrapolar a las plantas y a otros organismos, como también a fenómenos naturales y sociales.

En este punto es necesario tener claridad de que el objetivo es el diálogo, no es la imposición y supremacía de un tipo de conocimiento sobre el otro. Debe considerarse como una oportunidad para establecer puntos de encuentro y desencuentro en los que toman distancia, lo cual lleva a pensar y reflexionar sobre sus contextos de uso y aplicación de cada uno de ellos. Por ello es fundamental, tal y como plantea Aikenhead y Ogawa (2007), que los cuerpos docentes de ciencias naturales establezcan puentes entre sus propios sistemas de conocimientos, la mayoría de las veces eurocentrista, positivista y homogenizador, quizás por su formación tanto escolar como profesional inicial, incluso continuada, y otras formas de conocimiento. Este aspecto es fundamental, si se quiere establecer diálogo con otras formas de representación. En esa línea de pensamiento, Baptista y El Hani (2009) proponen que la etnobiología puede contribuir a ese diálogo entre culturas y diferentes tipos de conocimientos.

Retomando la metáfora del puente, en este texto se propone, a partir de los resultados obtenidos en una fase de un proceso investigativo de trabajo colaborativo con la docente donde se llevó a cabo el trabajo que dio origen a este texto, que el puente lo conforman y se sostiene en ambos tipos de conocimientos, pero es el lenguaje quien favorece la comunicación, es decir, el poder transitar de un lado al otro. Ahora bien, en ese diálogo y comunicación que se establece en la configuración y tránsito en la metáfora del puente, y retomando e interpretando los aportes de Cobern (1996), es pertinente señalar que la identificación y caracterización de los 
doi: http://dx.doi.org/10.15359/ree.22-2.1

URL: http://www.una.ac.cr/educare

CORREO: educare@una.cr

conocimientos alternativos no debe encaminarse a motivar, influenciar e invitar, por la parte docente, a que sus estudiantes cambien sus visiones de mundo y de la naturaleza misma, ya que es necesario comprender el conocimiento científico como una segunda cultura, con la que es necesario dialogar e intercambiar saberes. Por ello señala que no se trata de romper con las visiones del estudiantado, sino de ampliarlas y usarlas de acuerdo con los contextos. El mismo Cobern (2004), atendiendo las diferencias que plantea entre compresión y aprehensión, es enfático al señalar que el objetivo de la enseñanza de las ciencias es la compresión.

En ese sentido, es importante pensar que también, desde lo metodológico, se requieren cambios, se necesita pasar de una mirada única, homogenizante y de poder de la ciencia, la cual se ha establecido desde posturas epistemológicas, sicológicas o metodológicas universalistas de la ciencia, donde tiene gran relevancia el carácter individual y objetivo en la construcción del conocimiento; a la enseñanza y aprendizaje de las ciencias desde una perspectiva sociocultural y contextual, que se apoya en perspectivas antropológicas y sociológicas, las cuales enriquecen la mirada hacia considerar la enseñanza y aprendizaje de las ciencias como relaciones entre culturas (Cobern \& Loving, 2001; Molina, 2010).

Son diversos los trabajos que se han desarrollado o destacan la importancia de esta perspectiva. Sobresalen, entre otros, el de Hills (1989) para quien la enseñanza y aprendizaje de las ciencias naturales es una actividad de intercambio cultural al interior de una cultura o entre culturas. En esta misma línea es importante tener en cuenta lo que anota Wilson (1981), pues él resalta la importancia que tiene para la enseñanza de las ciencias el contexto cultural, ya que facilita un escenario para que se establezca la utilidad de lo que se enseña. Por esta misma vía, Cobern, \& Loving (2001) establecen que una visión de ciencia múltiple, es decir, donde convergen diferentes tipos de conocimientos, puede favorecer un efecto contrario y positivo al que la ciencia occidental ha generado en culturas no occidentales.

De esta manera destaca la importancia que tiene el contexto cultural, donde los procesos históricos, valores, y creencias de una comunidad son determinantes a la hora de proponer y desarrollar currículos diferenciadores y particulares, que tomen en consideración estas singularidades y reconozcan que las comunidades donde se insertan las escuelas poseen saberes tradicionales y ancestrales, los cuales tienen sentidos y significados diversos, que pueden ser enriquecedores por la clase de ciencias naturales al entrar en diálogo con los de la ciencia escolar, ya que los conocimientos científicos escolares se establecen y estructuran en la interacción con los conocimientos de las culturas estudiantil. Este aspecto se evidenció en el desarrollo de las actividades que se plantearon para estudiantes del grado sexto de la Escuela Rural San Lorenzo, y que fueron la génesis de las actividades que se proponen en este texto. 


\section{Implicaciones en la enseñanza de las ciencias naturales}

A continuación, se puntualizan tres implicaciones que espera tener este trabajo en el profesorado, particularmente en el de ciencias naturales. La primera está relacionada con la importancia que tiene comprender las relaciones entre la cultura y la educación, donde el contexto cultural tiene un papel central, pues es través de este que los individuos expresan y ponen en evidencia sus formas de relación, intereses, necesidades y saberes, entre otros aspectos (Molina, 2010). Este reconocimiento conlleva a reconfigurar el papel de la escuela, y dejar de verlo, como plantea Bolívar (2004), como un espacio unificador, igualitario, neutro, universal y extraterritorial, producto de una ideología de la modernidad, donde los medios de comunicación han tenido y siguen teniendo un gran poder.

La segunda, y desde el ámbito didáctico, el uso de diferentes estrategias para identificar y organizar los conocimientos faunísticos, o de otra índole, que tienen los niños y niñas de las escuelas rurales, significa darles valor a los llamados conocimientos alternativos, los cuales pueden tener un origen en los conocimientos tradicionales, científicos o científicos escolares, entre otros, y son el resultado de todo el conjunto de nociones y concepciones, las cuales abarcan presupuestos, creencias e interpretaciones que los niñas y niños tienen en un momento dado, entre otras cosas sobre los fenómenos científicos específicos (Cuéllar, 2009), y son la resultante de la relación que han establecido con miembros de su comunidad y con la naturaleza misma (Sepúlveda, 2003).

Y la tercera, es fundamental considerar que el conocimiento faunístico de una región es de gran importancia a la hora de planear estrategias de conservación por parte de la propia comunidad, incluyendo la escolar. Estos estudios pueden servir como punto base de investigaciones que pretendan establecer la situación y estado actual de la conservación de las especies en un espacio geográfico determinado, lo cual permite el diseño de medidas proteccionistas más acordes con el contexto cultural.

\section{Conclusiones}

A partir de los resultados del trabajo realizado con el estudiantado de sexto grado de una escuela del sector rural, además de las reflexiones que el autor hace del propio proceso de análisis de la experiencia desarrollada, como también de la escritura de este texto, a continuación, se puntualizan las siguientes conclusiones.

- Generalmente los currículos, y en este caso de ciencias naturales, han sido diseñados sin tener en cuenta el contexto escolar y sus particularidades; se ha basado en políticas educativas, que la mayoría de las veces obedecen a estándares internacionales, incluyendo los aspectos de tipo económico. 
doi: http://dx.doi.org/10.15359/ree.22-2.1

URL: http://www.una.ac.cr/educare

CORREO: educare@una.cr

- Es esencial el papel activo y decisivo del personal docente de ciencias naturales del sector rural a la hora de considerar e integrar al currículo, al aula y a su práctica docente estos conocimientos, la mayoría de las veces subvalorado, incluso invisibilizado. Es el propio personal docente quien debe considerar el contexto cultural (cosmovisión, costumbres y formas de organización, entre otros aspectos) de la escuela y de los miembros de su comunidad.

- Es fundamental que los docentes y las docentes interpreten, propongan criterios de organización y estructuración, con una visión más holística, integral y contextual del currículo de ciencias. Esto implica abrirse a otras lecturas sobre los diferentes estamentos y normas; como también empoderamiento en términos de decisiones y responsabilidad social.

- Muy de la mano con los aspectos anteriores, se propone que en esta tarea no se deje al personal docente del sector rural solo, sino que sea acompañado de docentes con mayor experticia en la investigación, lo cual favorece que se sienta parte de un equipo de trabajo y de una comunidad, en la que el trabajo colaborativo es la base de la interacción y del intercambio de experiencias.

- Teniendo en cuenta la experiencia del autor en este proyecto, las clases de ciencias también se pueden hacer sin equipos y laboratorios sofisticados. Para el caso que se propone el laboratorio es el propio contexto, o una parte de él.

- Esta forma de concebir la enseñanza de las ciencias, tomando como recurso fundamental los conocimientos tradicionales del estudiantado, en este caso los niños y las niñas del sector rural, favorece el desarrollo de actitudes más positivas hacia la ciencia misma, motiva al estudiantado a hacer preguntas, a reconocer la importancia del contexto cultural y a reconocerse como parte de un sistema biocultural.

\section{Referencias}

Aikenhead G. y Ogawa M. (2007). Indigenous knowledge and science revisited. Culture Studies of Science Education, 2(3), 539-620. doi: https://doi.org/10.1007/s11422-007-9067-8

Baptista, G. C. S. (2004). Etnozoología y la enseñanza de las ciencias biológicas. En E. M. C., Neto, M. Vargas (Coords.), Manual de etnozoología. Una guía teórico-práctica para investigar la interconexión del ser humano con los animales (pp. 148-162). Valencia: Tundra Ediciones. Recuperado de https://www.researchgate.net/profile/Erendira Cano-Contreras/ publication/310507403 Cap 4 Cosmovision y Etnozoologia Cap final Etnobiologia para que para quien/links/5830b36608aef19cb817f904/Cap-4-Cosmovision-yEtnozoologia-Cap-final-Etnobiologia-para-que-para-quien.pdf 
Baptista, G. C. S. (2007). A contribuição da etnobiología para o ensino e à aprendizagem da ciências: Estudo de caso em uma escola pública no estado da Bahia (Master Thesis). Universidade Federal da Bahía, Salvador, Bahia. Recuperado de https://repositorio.ufba. br/ri/bitstream/ri/15805/1/Geilsa\%20Costa\%20Santos\%20Baptista.pdf

Baptista, G. C. S. y El-Hani, C. N. (2009). The contribution of ethnobiology to the construction of a dialogue between ways of knowing: A case study in a Brazilian public high school. Science and Education, 18(3), 503-520. doi: https://doi.org/10.1007/s11191-008-9173-3

Bolívar, A. (2004). Ciudadanía y escuela pública en el contexto de diversidad cultural. Revista Mexicana de Investigación Educativa, 9(20), 15-38. Recuperado de http://www.bantaba. ehu.es/lab/files/view/Ciudadan\%c3\%ada\%5fy\%5fescuela\%5fp\%c3\%bablica\%2epdf?revi sion $\% 5$ fid $=67422 \&$ package $\% 5$ fid $=67398$

Clavijo, M. (2009). Patrimonio zoocultural: El mundo animal en las expresiones tradicionales de los pueblos. En E., Neto, D. Fita y M. Clavijo (Coords.), Manual de etnozoología. Una guía teóricopráctica para investigar la interconexión del ser humano con los animales (pp. 118-144). Valencia:TundraEdiciones.Recuperadodehttps://www.researchgate.net/profile/Erendira Cano-Contreras/publication/310507403 Cap 4 Cosmovision y Etnozoologia Cap final Etnobiologia para que para quien/links/5830b36608aef19cb817f904/Cap-4Cosmovision-y-Etnozoologia-Cap-final-Etnobiologia-para-que-para-quien.pdf

Cobern, W.W. (1996). Constructivism and non-western science education research. International Journal Education, 18(3), 295-310. doi: https://doi.org/10.1080/0950069960180303

Cobern, W. W. (2004). Apples and oranges: A rejoinder to Smith and Siegel. Science \& Education, 13(6), 583-589. doi: https://doi.org/10.1023/B:SCED.0000042856.33782.7b

Cobern, W. \& Loving, C. C. (2001). Defining "science" in a multicultural world: Implications for science education. Science Education, 85(1), 50-67. doi: https://doi.org/10.1002/1098237X(200101)85:1<50::AID-SCE5>3.0.CO;2-G

Costa, E. M., Santos, D. y Vargas, M. (Coord.). (2009). Manual de etnozoología. Una guía teóricopráctica para investigar la interconexión del ser humano con los animales. Valencia: Tundra Ediciones. Recuperado de https://www.researchgate.net/profile/Erendira Cano-Contreras/publication/310507403 Cap 4 Cosmovision_y Etnozoologia Cap final Etnobiologia para que para quien/links/5830b36608aef19cb817f904/Cap-4Cosmovision-y-Etnozoologia-Cap-final-Etnobiologia-para-que-para-quien.pdf

Cuéllar, Z. (2009). Las concepciones alternativas de los estudiantes sobre la naturaleza de la materia. Revista Iberoamericana de Educación, 50(2), 1-10. Recuperado de http://www. rieoei.org/deloslectores/2834Cuellar.pdf 
doi: http://dx.doi.org/10.15359/ree.22-2.1

URL: http://www.una.ac.cr/educare

CORREO: educare@una.cr

Delors, J. (Presidente). (1996). Los cuatro pilares de la educación. En J. Delors (Presidente), Informe a la UNESCO de la Comisión Internacional sobre la educación para el siglo XXI. La educación encierra un tesoro (pp. 91-103). Madrid: UNESCO. Recuperado de https://www. uv.mx/dgdaie/files/2012/11/CPP-DC-Delors-Los-cuatro-pilares.pdf

Enciso, S. y Molina, A. (2009). La resolución de problemas y sus interrelaciones con el contexto cultural: Determinación de referenciales para la formulación de una propuesta de formación inicial de licenciados en química. Enseñanza de las Ciencias, Extra, 3416-3419. Recuperado de http://www.raco.cat/index.php/Ensenanza/article/view/294698/383232

George, J. (2001). Culture and Science Education. A look from the developing world. Recuperado de http://www.actionbioscience.org/education/george.html

Gobierno de Colombia, Asamblea Nacional Constituyente. (1991). Constitución política de Colombia. Recuperado de http://es.presidencia.gov.co/normativa/normativa/ Constitucion-Politica-Colombia-1991.pdf

Hernández, R. (2014). Contexto cultural y currículum en la enseñanza de las ciencias (Capítulo 7). Enseñanza de las ciencias y cultura: múltiples aproximaciones), 145-163. Recuperado de http://die.udistrital.edu.co/sites/default/files/doctorado ud/publicaciones/contexto cultural y curriculum en ensenanza ciencias.pdf

Hernández, R. (2017). El contexto cultural en las prácticas educativas de profesores de ciencias del sector rural: Perspectivas para el modelo de formación por cambio didáctico (Tesis doctoral). Universidad Distrital Francisco José de Caldas, Bogotá, Colombia. Recuperado de http:// repository.udistrital.edu.co/bitstream/11349/6455/1/HernandezBarbosaRubinsten2017.pdf

Hills, G. L. C. (1989). Students' "untutored" beliefs about natural phenomena: Primitive science or commonsense? Science Education, 73(2),155-186. doi: https://doi.org/10.1002/ $\underline{\text { sce. } 3730730204}$

Jegede, O. J. (1995). Collateral learning and the eco-cultural paradigm in science and mathematics education in Africa. Studies in Science Education, 25(1), 97-137. doi: https:// doi.org/10.1080/03057269508560051

Lopes, A. R. C. (1999). 4 Pluralismo cultural em políticas de currículo nacional. En A. F., Moreira (Org.), Currículo: Políticas e prácticas (10ª ed., pp. 59-80). Campinas: Papirus.

Margalef, R. (1998). Ecología (9a ed.). Barcelona: Omega.

Molina, A. (2010). Una relación urgente: Enseñanza de las ciencias y contexto cultural. EDUCyT, $1(1), 1-12$. 
Molina, A., Martínez, C. A., Mosquera, C. J. y Mojica, L. (2009). Diversidad cultural e implicaciones en la enseñanza de las ciencias: Reflexiones y avances. Revista Colombiana de Educación, 56, 106-130. Recuperado de http://www.redalyc.org/pdf/4136/413635250006.pdf

Molina, A., Mojica, L. y López, D. (2005). Ideas de los niños y niñas sobre la naturaleza: Estudio comparado. Revista Científica 7, 41-62. Recuperado de http://die.udistrital.edu.co/sites/ default/files/doctorado ud/publicaciones/articulos en revistas nacionales indexadas ideas de los ninos y ninas sobre la naturaleza estudio.pdf

Sepúlveda, S. (2003). A relação entre ciência e religião na alumnos protestantes de alumnos de formação em ciências biológicas (Master Thesis). Universidade Estadual de Feira Santana. Brasil, Salvador.

Wilson, B. (1981). The cultural contexts of science and mathematics education: Preparation of a bibliografic guide. Studies in Science Education, 8, 27-44. doi: https://doi. org/10.1080/03057268108559885 